Journal of The Magnetics Society of Japan Vol. 13, Supplement, No. S1 (1989)

(C) 1989 by The Magnetics Society of Japan

\title{
MAGNETIC RECORDING RETROSPECTIVE AND PROGNOSIS
}

\author{
C. Denis MEE \\ IBM Corporation, San Jose, California 95193
}

\begin{abstract}
Consistent advances in magnetic recording products and applications over several decades have resulted from increases in recording density and bandwidth using the same basic design of recording components. Now, new components and recording methods are receiving major development emphas is and are candidates to extend performance beyond today's product achievements. The key winning and losing magnetic recording components and techniques are reviewed and the new challenging designs are considered for future applications

Magnetic heads, media, and recording methods have evolved in different ways to extend recording performance and density for data storage, and for consumer video and audio recording applications. In particular, the development of new components for consumer recording systems may provide lower cost technology for data storage applications. Some examples of successful application of components to different recording systems will be described.
\end{abstract}

\section{INTRODUCTION}

Over the last decade, a very large effort has been made to understand the magnetic recording capabilities of perpendicular recording. It is the only novel recording technique to be seriously pursued during the past 25 years, when magnetic recording has achieved unimpeded growth in both consumer and data storage applications. Perpendicular recording technology has succeeded in demonstrating very high-density storage capability with newly developed heads and media design for magnetic tape, flexible and rigid disk devices.

In order to understand how perpendicular recording fits into the ongoing development of magnetic recording technology, it is instructive to assess the momentum of the current technology and where are the most difficult challenges. This assessment will include what other technologies offer relief from these magnetic recording challenges and what other strengths or weaknesses accompany those technologies.

We will examine the progress in magnetic recording technology through its major components, heads, media, and recording methods. Other important technological advances such as low-cost electronics, mechanical accessing of heads and media, and signal coding and error control are of vital importance in the development of recording technology. These technologies are mostly common to the current recording technologies and to perpendicular recording and are not covered here in any detail.

The rating of component advances in the past and contenders for the future is divided into five categories (Fig. 1). The first category includes components that have had successful product application for the last 25 years--known as the "veteran stars" (shown in Fig. 1, clockwise, from the top left-hand corner). Next comes the "new stars" which are components with major product application in the last 25 years. Thirdly, we will consider the "contenders" which are components or techniques with major development activity, but are not yet in extensive product application. This is followed by components with very selected applications, i.e., "niche components," and, lastly, those few product components or techniques that have been discontinued or have significantly diminished application.

\section{MAGNETIC RECORDING MEDIA}

Looking at the evolution of magnetic recording technology from the point of view of the recording media, the challenge has been to preserve reliable interfaces with recording heads. Mechanical interactions have become increasingly aggressive due to increased head-media velocities and decreased spacings. At the same time, the operating environment for the recording medium became more and more constrained due to miniaturization of drives but with less environmental contro1. The requirement for 
reliable recording under these increasingly adverse conditions has played a major role in determining the relative successes of the many possible formats, packages and constituents of modern recording media.

Figure 1 illustrates the history and trends in recording media evolution. Advances of three types have taken place simultaneously, driven by the development needs of audio, video and data recording. First, as shown in Figure 1 , media in the form of disks and tapes have succeeded over a11 other formats in a11 major magnetic recording application areas. On the other hand, drums, strips, belts, and cards were eventually discarded due to poor reliability or cumbersome form factor. Convenient removability of the recording medium from a drive has also had a major influence on the success or failure of different formats. A new media format that has emerged during the demise of strips, belts, and cards, is the flexible disk. It is a low-cost data interchange medium and is used in all personal computers. While the storage capacity of flexible disks is being extended for data storage, this inexpensive and reliable medium is finding additional applications in cameras and lap-top computers using twoinch-diameter disks. A more expensive design of flexible disks, with improved mechanical stability, has been introduced with rigid rings supporting the outer and inner disk circumferences. This stretched surface disk is a potential challenge to the rigid disk, and claims to have a durable head-media interface at very low flying heights. However, rigid disks continue to improve in performance and cost and have not been seriously challenged. Another type of mechanically stabilized flexible disk uses a stationary plate in close proximity to the rotating disk to provide a reliable headmedium interface at higher rotational velocities than the simple flexible disks. This Bernoulli disk has been a successful niche product. Its future may be challenged by much higher capacity erasable-optical disks.

Other media formats which continue to be successful in specific niche applications are magnetic stripes and sheets. The applications, such as magnetic cards or motion picture films, rely on the convenience of recording a magnetic stripe coupled with

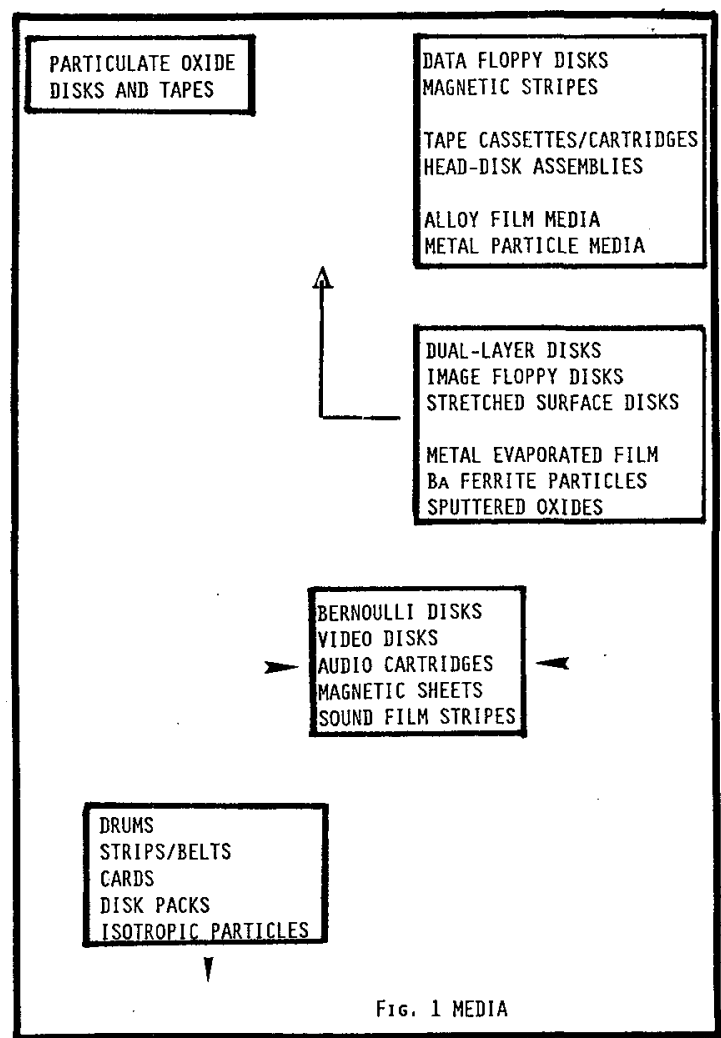

adequate storage durability. They do not exploit the high-density recording capability developed in disk and tape formats.

Next, we consider the impact of the development of reliable media containers in expanding the use of magnetic recording products in audio, video, or data storage applications. In the early days of printing, Gutenberg's invention of movable type did not lead to widespread use of the printed word until about 50 years later when Aldus Manutius (a Venetian) developed the portable book. This packaging of the printed page provided the successful distribution medium. In the same way, the application of the open-tape reel was restricted, and really widespread use of consumer magnetic tape awaited the development of the tape cassette or cartridge. Since the 1960 s, reel-to-reel cassettes have enjoyed ever increasing application in audio, video and data recording, enabling the tape consumer to use the highest recording densities in inexpensive and conveniently 
sized protective packages. The continuing capability of recording technology to increase areal density has led to smaller and more convenient packages, opening up new consumer applications such as movie cameras, lap-top computers, and digital audio. It is consumer applications such as audio and video tape recorders that have often provided the opportunities for advanced media and media packaging designs which subsequently migrate to other applications such as data storage.

In the rigid-disk data storage business, a successful container for the disk pack started with the introduction of the Winchester technology in the early 1970s. Both the media and the heads had to be sealed in the container in order to control contamination to a level sufficient to attain very high recording densities. This non-removable head-disk assembly quickly became the predominant package for all disk storage devices, displacing the removable disk pack almost completely today.

The evolution of the flexible-disk container to a hard-plastic shell from the original flexible jacket has provided adequate protection for the more vulnerable highdensity flexible disks. Use of this type of container has migrated to removable optical disk applications for data storage.

Thus, from the above examples, it is seen that magnetic recording disk and tape media are no longer handled without some protective container. In general, media containers have evolved separately for consumer and data recording applications since greater protection is required for data storage.

The third area of consistent improvement and advancement is in the recording medium itself. It is remarkable that nearly all of the magnetic materials in film or particulate form in use today were under deve1opment twenty years ago. A notable exception is the cobalt-chromium film medium which is the primary film disk recording medium today; this material was first used in perpendicular recording in the late 1970s.

The veteran oxide particle medium continues to be extensively used in all major magnetic recording applications today. The fundamental advantage of having separate materials in a coating to provide the mag- netic storage and mechanical characteristics has allowed a sustained period of media improvement using basically the same manufacturing processes and skills. This continued momentum of improvement of disk and tape media has been fueled by introductions of improved dispersions of smaller particles with ever increasing magnetic hardness. The original acicular iron oxide particles have been improved with cobalt additives to provide a large range of coercivities. Perhaps the only victim in this evolution has been the attempt to introduce isotropic particles with cobalt additives, which had insufficient stability for the thermal and mechanical stress conditions of today's recorders. On the other hand, acicular chromium dioxide particles have succeeded in both consumer and data tape applications having sufficient storage stability combined with competitive recording performance. Oxide recording media will also contend for future applications with the development of small barium ferrite recording particles with the desired magnetic hardness. The double attraction of barium ferrite is that it is usable for both longitudinal and perpendicular recording.

Despite the success of recording system designs to continuously increase recording densities in the past, the need for higher magnetization media has steadily increased in order to provide adequate signals in inductive recording applications. The magnetic alloy powders and films have the desired magnetic properties but have, until recently, been too expensive and difficult to produce with adequate stability. Now, consumer tape applications again are the leading applications for new metal particle tapes with high magnetic moment and correspondingly high coercivities. Adequate passivation of metal powders has been achieved and it is expected that they will be applied to all flexible media products.

on the other hand, a bigger change is taking place in rigid disk recording media. For the first time, the storage medium design is not following the consumer products lead. Instead, the exploitation of magnetic alloy media is taking place in thin film disks. Alloy-film media with protective overcoats are now preferred for high-density disk recording and are already equal to oxide particulate media in production volume. 
They have been designed for closer flying in rigid disk applications and are superior in magnetic properties. They will be a leading contender for future high-density designs which might require discrete magnetic tracks or mu1tiple layers, as used in perpendicular recording. Alloy-film rigid-disk media have had to overcome two majox technical challenges to find successful product applications. For many years, the very thin metal film was not adequately protected from head-media contacts, and also the sticking together of the smooth surface heads and disks was a problem when the disks were not spinning. In addition, the magnetic layer itself did not record accurately the sharp transitions between recorded bits. Large local demagnetization fields at transitions could disturb the local magnetization which, in turn, increased the disk noise. Perpendicular recording avoids this latter problem. However, both problems have been effectively solved in longitudinal recording media with a combination of overcoats, lubricants, disk topography control to improve mechanical performance, and disk microstructure to provide improved magnetic stability. With these problems solved, and the emergence of large-scale manufacturing equipment for film deposition, low-cost high-performance alloy film disks are beginning to outsell oxide particle or oxide film disk designs.

The most challenging design for a magnetic alloy recording medium is the alloy film tape. Under development for the last 30 years, this format has also reached consumer product applications recently. Earlier designs with plated media gave way to direct evaporation of the alloy onto the plastic substrate. This low-cost process is capable of producing multi-layer films with recording performance superior to any particulate recording tape. Again, the pacing item in the development of film tape has been the design of an adequate mechanical interface. In the future, film tape can be a contender for all flexible media applications.

\section{HEADS}

One of the most remarkable facts about magnetic recording heads is that the original concept of an inductive ring head has been applied in every successful magnetic recording device built in the last 50 years (see Fig. 2). This head design performs all of the required functions of writing, reading, erasing and overwriting and, in many applications, a single head element provides all the required functions. This feature has been a great advantage for subsequent development of lightweight accessing heads. It is, of course, an advantage which is shared by recently developed pole-heads for perpendicular recording.

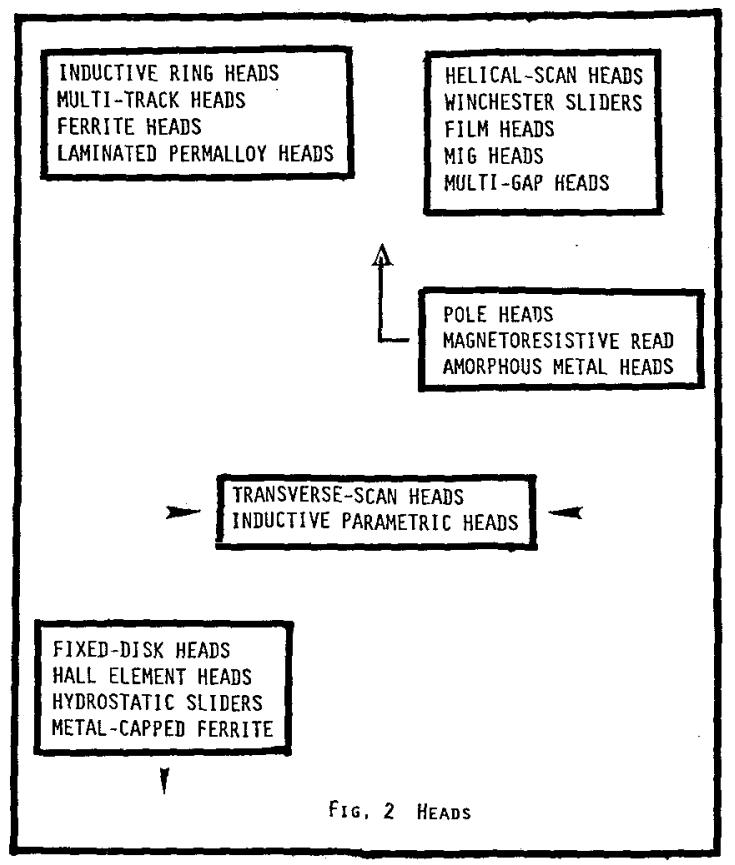

Inductive ring-head applications have evolved with the availability of new magnetic materials that provide higher fields at higher recording bandwidths. The requirements of higher recording densities have been to control gap and track geometry while maintaining adequate resistance to wear and environmental effects. Inevitably this has led to combinations of magnetic films and bulk magnetic materials in head structures. Many designs of the inductive ring head have emerged over the years to accomplish improvements in cost and performance. The most expensive design direction has employed multitrack heads for high-bandwidth applications. This costly approach spurred the development of batch 
fabrication techniques for heads such as thin film head designs.

Future design trends in magnetic heads will continue to use the same types of magnetic materials as are under development for the inductive ring head. Additional hard magnetic materials may be needed in future heads to help control their magnetic domain structure. Perpendicular pole heads can use all of the materials developed for inductive ring heads and can also be fabricated with similar process steps.

Two major development trends have emerged for disk and tape recording applications which push the design of heads beyond the bulky fixed heads used for early audio and data recorders. First, for all disk recording, an accessing head is required which is very $1 \mathrm{ightweight}$ and usually a single-track element. This requirement spurred the design of air-bearing sliders with small read-write heads incorporated at the proximity point to the recording medium. Secondly, for tape recording, the requirement for high bandwidth at modest tape speeds has been satisfied with a scanning single-track head for most applications. This development has challenged the conventional stationary multitrack head. Starting with video recording, then audio and now data recording, helical scanning heads have considerably widened the fields of application for magnetic recording and are competitors for a11 tape applications.

Although laminated permalloy heads are still produced in large quantities throughout the world, the champion of the veteran head materials is manganese or nickel zinc ferrite which has been used extensively in al1 magnetic recording devices during the last 25 years. Its original attributes of a long-wearing stable material, which could be formed into poles with we11-defined magnetic head gaps, were combined with materials developments that extended the bandwidth. Today, the limitation of the inherently low magnetization of ferrite have necessitated the complication of adding high-magnetization alloy pole-tips. Even though this modification involved technical challenges such as the unwanted introduction of secondary gaps, the so-called metal-ingap head has found application in all highdensity recording devices today. Ferrite heads have also been adapted to provide multiple gap structures such as the tunnel-erase head, used in large volume for flexible disk heads. In this application, due to poor tracking, auxiliary heads are required to erase the track at the side of the head to reduce off-track noise.

The only radically new fabrication technique for magnetic heads, during the last 20 years or so, has been the introduction of the film head in the $70 \mathrm{~s}$ in which the magnetic circuit, coils, insulators, and gaps are all deposited films. Design of film heads has evolved from early singleturn multitrack heads to multi-turn designs with up to at least 50 turns. Film heads with finite pole-tips have been designed for high-bandwidth and high-resolution readwrite elements, for rigid disk files, where they have a major application today. Mu1titrack film heads with separate read and write elements have been applied to digital audio and data recording, but find their major application in data tape recording today. In this application, a film inductive writing head can be used in conjunction with a film magnetoresistive reading element. This combination is a powerful design for advanced recording applications since it combines the high output of a magnetoresistive reading element with an inductive writing head optimized for high recording fields. This is really the second generation for film head designs and it promises much more design optimization flexibility than is obtained with the compromise single write/read element. For instance, the writing track width may be made wider than the read track, thus easing the servo requirements for a high track density disk or tape drive. Secondly, new high-magnetization materials may be used in the write portion of the head to provide recording fields sufficient for the very high coercivity media required for future advances in recording density.

Three classes of magnetic materials are being pursued in the quest for satisfactory high-magnetization materials. Ternary a1loys with significant cobalt content or aluminum-iron- silicon alloys with additives have demonstrated higher magnetization than perma11oy, and have been developed for head poles. Sputtered amorphous alloys 
of many compositions have been developed and can combine high magnetization, low coercivity and good wear characteristics. Finally, laminated films are under development which exhibit the same desirable characteristics. Laminated amorphous films and insulation $f i l m s$ have been developed to reduce eddy current losses in high-frequenty head applications. With progress in several approaches to high-magnetization soft magnetic properties, the choice may well be based on compatibility with head processing and subsequent long-term stability in the head operating environment. Again, poleheads used for perpendicular recording can be built using the same thin film materials and technology already developed for longitudinal recording heads.

Challenges for thin film head designs center around the increasing difficulty of controlling the magnetic domain structure of the very small magnetic elements required for high recording densities. In the case of inductive head designs, unwanted domain wall motion causes erratic magnetization reversals affecting both the head sensitivity and its noise level adversely. Although Dr. Barkhausen first observed this noise in magnetic materials in 1919, the problem has not been eliminated and it still plays a role in magnetic head performance today. In fact, in perpendicular recording it is also a factor in the performance of the recording medium.

Magnetoresistive reading elements are also prone to Barkhausen noise both in the reading element and its accompanying shields. Controlling these domain structures has added significant complication to magnetoresistive head designs where additional hard or exchange-coupled magnetic films are required to enforce a desired rotational magnetization mode in the reading element. Today, magnetoresistive reading heads have been candidates for audio recording applications and have found product application in data tape storage devices. Due to the use of an active, thermally sensitive and noisy element requiring additional magnetic bias, MR elements are complicated and have special design requirements to avoid wear, corrosion and electrical disturbance. There is some hope that MR-head inductive-head combinations can be simplified in the future by integrating the read element into the gap of the writing head. This will provide for easier read-write track alignment and will avoid misalignment due to head skewing. However, the MR element is then subjected to the full writing field disturbance, thus aggravating the domain control problem. MR heads are very sensitive, with high outputs independent of tape velocity, and will extend magnetic recording performance beyond the capability of inductive heads. They can be expected to be applied in disk as well as tape systems as their cost and design are optimized. Despite the complicated structures of MR heads requiced to control high track and bit density designs, the application to tape drives, being relatively wide tracks today, is a much simpler design problem. This has led to simple multitrack designs for tape heads which have become preferred over the more cumbersome inductive write and read combinations.

\section{Tape Head Design Trends}

The most successful new head design of the last 20 years or so for tape application has been the helical-scanning drum with transformer-coupled read-write head elements. This design has superior track den= sity capability, incorporating continuous head positioning and can have a very wide range of surface velocities leading to high-bandwidth-per-head element. With the highest density recording capability, the scanning head is able to compete effectively for all serial recording applications from the 1Gb/s digital television requirement (using multipole heads) to the $3 \mathrm{Mb} / \mathrm{s}$ bandwidth of digital audio recorders. Head-tape wear rates are being continuously improved with the introduction of new materials such as soft amorphous poles for the ferrite heads, and thus the advantages of high-coercivity metal particle tapes can be used for high-density recording with scanning heads. The scanning head has challenged the traditional multitrack stationary head for many applications. The last major application, not yet challenged completely, is in data storage. Helical scanning heads are already being applied in instrumentation recorders and are entering the low-performance data 
storage areas. In applications whore rapid cassette accessing is required along with high data rates, the multitrack head is still the most popular design.

\section{Rigid Disk Head Design Trends}

Disk files are usually designed with multiple recording surfaces and sometimes with more than one head per surface. Thus, disk heads are required in relatively large volumes and a simple low-cost design for the accessing head carrier is required. This requirement was fulfilled 16 years ago with the introduction of a taper-flat slider known as the Winchester slider. This simple design, originally fabricated with magnetic ferrite, was integrated with a ferrite core. Today the Winchester slider is used in a11 rigid-disk files. It has evolved from a11ferrite designs, with two or three rails, to composite designs with ceramic slider and ferrite or film head elements attached. A11 of these designs are currently used in products, the all-ferrite (monolithic) head still has the highest volume usage followed by composite ferrite and film heads. Cost has much to do with choice of design. Other advances in Winchester slider design center around reducing the volume of the slider and this has allowed disks to be packed close together. Thus, both tape and disk recording systems are being we11 served by extendable head accessing technologies and a variety of advanced head elements with improved magnetic and mechanical properties. The major technical challenge continues to be the design of durable and reliable headmedia interfaces which will require matched development of head and disk materials.

Numerous head designs of the past have been discarded due to being superseded by simpler designs with competitive function. In addition to fixed multitrack heads for disks, files, and metal-capped ferrite heads for tape recording which were in product usage in the past, many more complicated designs never achieved major product application. This includes, for instance, magnetic scanning heads and electron or optical beam recording heads.

\section{RECORDING TECHNIQUES}

With the expansion of magnetic recording into disk and tape recording applications at ever increasing recording densities, there has been a steady evolution of component technology to deal with the concommitant deterioration of the recorded signal. Part of this advance has required innovative technology to discriminate the wanted from the unwanted signal. Servo technology to position the head over the wanted recording track has required advances in head or media positioning devices along with accurate position-sensing signals. Signal processing has been developed to improve the discrimination of the information being read from other information recorded upstream or downstream along the track. In digital recording, exror detection and correction methods have been advanced significantly to correct the signals which are corrupted sufficiently to cause a reading error. Excepting these advances in signal recovery techniques, there has been remarkably little introduction of fundamentally different recording techniques over the last two or three decades.

As indicated in Figure 3, longitudinal magnetic recording using the three recording methods developed at least 25 years ago remains the primary recording technology in products today. First, direct recording with ac bias is the mainstay of consumer audio tape recording and is widely used for instrumentation tape recording. It has been adequate for the quality required and is highly economical in tape usage. The signal-to-noise and recording density achievable has improved with the introduction of higher quality tape designs and higher resolution head designs. Secondly, FM recording, without bias, offers wide-band recording down to DC response. Amplitude signal losses are minimized and efficient packaging of the video signal across the recording band made this the recording method of choice for video recording. It has also been applied in instrumentation recording. Thirdly, saturation digital recording without bias has been applied to all forms of data recording due to its superior reliability and correctability. Initially the least economical in storage density, digital recording is now achieving such high densities with reliability that it is 
spreading to al1 applications for magnetic recording. Digital audio recording is an accomplished product technology and digital video recording is entering the product arena.

It is appropriate here to recognize a recording method, called azimuth recording, that has made a significant contribution to recording density improvement in consumer video and audio recording, and also in flexible disk recording. By recording adjacent tracks with heads whose gaps are at an angle to each other, it is possible to reduce the sensitivity to adjacent recorded tracks. This sensitivity is wavelengthdependent and is tuned differently depending on the application. In consumer video recording it has been possible to eliminate guard bands between tracks. The playback tracking margin is significantly improved for digital tape recording applications such as professional digital video recorders and digital audio recorders.

A number of recording methods have either become very specialized in their application or have been discarded altogether; some examples are listed in Fig. 3. However, contact duplication of recordings has had transient interest on and off for many years. So far, direct drive-to-drive duplication of video recordings has proved to be a reliable, high-quality cost effective technique. With the prospect of an ever larger duplication market for digital audio tapes, contact recording at several hundred times normal speed is under development again. Earlier thermal transfer techniques have been discarded in favor of ac field anhysteretic magnetization of a barium ferrite slave tape. A high coercivity metal. particle tape is used as the mirror master recording.

The two new recording techniques contending for future applications are perpendicular recording and thermomagnetic recording. This conference is a milestone in the assessment of the evolution of the potential of perpendicular recording. The purpose of this paper is to describe the technology environment that perpendicular recording is entering. First, it is clear that pexpendicular recording uses component designs that are highly compatible with the technology and manufacturing trends of cur-

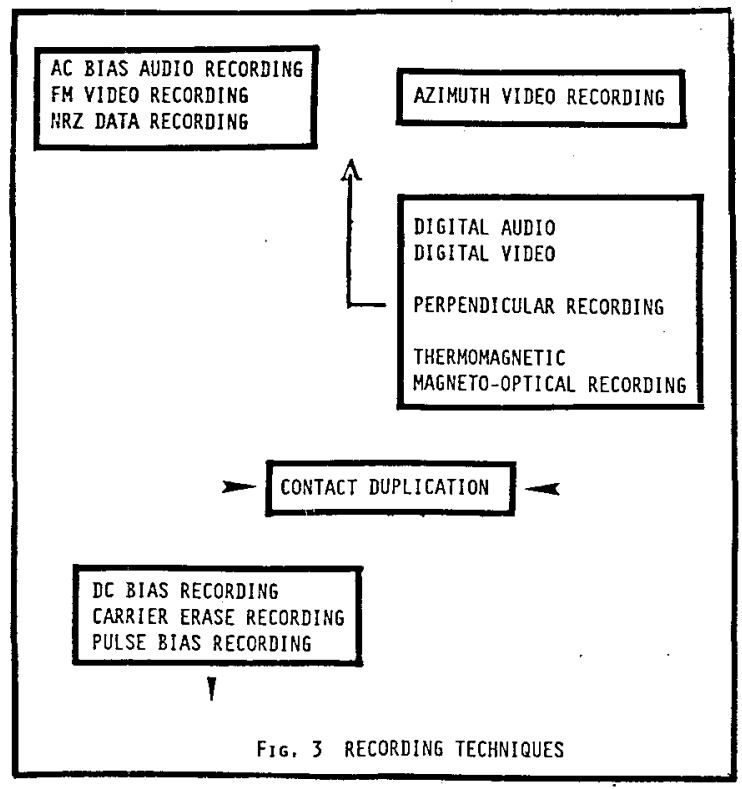

rent recording components. It can inherit the fabrication processes and the new materials that are under development for disk and tape applications. It can be designed to provide "drop-in" components for the present devices using channels similar to existing designs. However, the timing of the entry of perpendicular recording is not clear. At present, the incumbent longitudinal recording technology is anticipated to provide continued, if not accelerated, storage density increase over the next decade. Significant challenges center around the necessity to provide smaller head-medium separations and reliable magnetic storage in smaller and smaller volumes. Nevertheless, it seems that the historical doubling of areal density every two to three years will at least be maintained. Support for this thesis comes from the promise of very sma11 magnetic particles or crystallites in film media to maintain low media noise, plus higher sensitivity heads such as the magnetoresistive head to provide larger reading signals. Both perpendicular and longitudinal recording will benefit from improvements in interfaces, head and media components, track-following improvements, and better signal processing techniques. Since it is 
not known today where or when serious problems will occur in the advancement of highdensity recording, changes in the opportunity for perpendicular recording are difficult to anticipate.

The most challenging technology hurdle in disk or tape recording is the requirement to produce ever closer spacings at the head-media interface without engendering problems of head crashes or head-media stiction and wearing. Because of this, any high-resolution read/write technology offering fundamental relief from this problem is a candidate to be considered for future storage devices. In this respect, thermomagnetic writing and magneto-optical reading with a laser beam transducer is a viable candidate for magnetic recording applications. Today, this technology is supported by very extensive industrial evelopment activity and numerous first-generation drives are under development with some products already in existence. Will this technology evolve at a rate that will make it the superior method for high-density for magnetic storage in the future?

Magneto-optical storage technology was promoted for data storage for the first time about 25 years ago. It died because inadequate signal-to-noise ratios were obtained from polycrystalline films and laser diodes lacked the power and wavelengths required to effectively read and write with those films. In 1980, amorphous films were introduced for thermomagnetic recording and magneto-optical reading using a GaAs laser as the beam source. Over the last eight years, signal-to-noise ratios have been doubled by the advances in media composition, optical enhancement layers, substrate improvements and differential detection methods. Areal densities of 400-600 $\mathrm{Mb} / \mathrm{sq}$.in. have been achieved on disks with data rates typically in the low $\mathrm{Mb} / \mathrm{second}$ range. Optical heads are complicated and bulky today and, therefore, moderate access times of $30-60 \mathrm{~ms}$ are obtained in the firstgeneration products. This level of technology is not competitive with today's DASD but it offers very high-density storage and removable media.

Steady improvements can be expected in the future in density, access times and data rates with the development of shorter wavelength lasers, multiple lasers, simplified optics, high-bandwidth servos and better signal processing. Media performance will continue to improve as manufacturing techniques are upgraded. Even so, head-on competition with DASD will remain difficult for magneto-optical storage with the improvements anticpated for DASD, especially in volume storage density, being difficult to challenge. The most difficult problems for optical storage in DASD applications are designing an optical head with a low profile to allow disks to be stacked close together, and secondly the development work required to provide a direct overwrite capability at DASD data rates.

The more natural applications for optical storage are to exploit the removable media feature either as a very high-density removable-disk storage device or as a magnetic tape replacement offering improved performance. Even in these areas, magnetic recording devices will continue to provide more storage at lower cost and optical storage will also be required to evolve with low-cost designs for heads and media. Overa11, magneto-optical disk storage technology will advance in many ways analogous to magnetic recording over the last 10-20 years. Heads, media, channels, servos and actuators are all under intensive development. There are even some competing optical storage media (e.g., phase change) that can alleviate some of the engineering challenges of magneto-optical recording (e.g., head simplicity, direct-overwrite), but other challenges can be more severe in these media - In this young technology there is a healthy set of component design opportunities. Nevertheless, some of the prime attributes of magnetic recording--volume storage density of disks and tapes, high data rate per transducer and fast access-will provide a very difficult target for optical storage to challenge across the board. 